\title{
Die Degrowth-Bewegung und die Gewerkschaften
}

\author{
NORBERT REUTER
}

\section{Wachstumskritik und ökologische Krise}

Die Kritik am wirtschaftlichen Wachstum hat derzeit Konjunktur. Dies kann auch nicht verwundern, gilt „Wachstum“ doch gemeinhin als zentraler Auslöser globaler Probleme wie Erderwärmung, Anstieg der Meeresspiegel, Umweltkatastrophen, Artensterben und Extremwetter. Nach Angaben des aktuellen Living Planet Report 2012 des WWF ${ }^{1}$ benötigt die Menschheit, wenn sie so weiterlebt wie bisher, bis zum Jahr 2030 zwei Planeten, um den Bedarf an Nahrung, Wasser und Energie zu decken. Bis zum Jahr 2050 wären es sogar fast drei. Die Hauptverursacher sind ebenfalls bekannt: die fortgeschrittenen Industrieländer. Sie alle weisen einen ökologischen Fußabdruck ${ }^{2}$ auf, der weit über dem Weltdurchschnitt liegt. Um eine globale Umweltkatastrophe zu verhindern, sind vor allem Länder wie Dänemark, die USA, Belgien, Australien, Kanada, Niederlande und Irland, die zu den Top 10 im Ranking des ökologischen Fußabdrucks gehören, besonders gefordert. Deutschland gehört mit Platz 30 unter 150 erfassten Ländern ${ }^{3}$ zum oberen Fünftel.

Die hieraus sich ergebende Verantwortung der reichen Industrieländer ist Grundlage einer größer werdenden politischen wie zivilgesellschaftlichen Bewegung, die eine umfassende Wende in der wirtschaftlichen Entwicklung fordert. Dabei spielt die erwähnte Wachstumskritik eine herausragende Rolle. Gelegentlich scheint es so, als ob die Beantwortung der Frage „Wie hältst du es mit dem Wachstum?" darüber entscheidet, ob jemand als Mitkämpfer oder Gegner wahrgenommen wird. Vor diesem Hintergrund haben es beispielsweise die Gewerkschaften schwer, als Mitakteur einer sozialökologischen Wirtschaftswende akzeptiert zu werden, gelten sie doch gemeinhin als ebenso unkritische wie mächtige Befürworter wirtschaftlichen Wachstums.

\section{Wachstum des Bruttoinlandsprodukts - Indikator wofür?}

Dabei ist Wachstum - sofern hiermit wie in aller Regel das Wachstum des Bruttoinlandsprodukts (BIP) gemeint ist ein denkbar schlechter Indikator, um Aussagen über Nachhaltigkeit und die sozialökologische Qualität der Entwicklung machen zu können. Im Kern lassen sich hierfür drei Gründe anführen:
(1) Große Teile der tatsächlich erbrachten Leistung werden im BIP gar nicht erfasst, weil sie nicht marktförmig organisiert, erbracht und getauscht werden. Würden unbezahlte Tätigkeiten in privaten Haushalten (vor allem die sogenannte Sorge- oder Care-Arbeit), in politischen Parteien, Gewerkschaften, Kirchen etc. oder in Ehrenämtern bewertet und erfasst, läge das gemessene BIP in Deutschland um über ein Drittel höher, ${ }^{4}$ ohne dass die Realität hiervon berührt würde. Damit bleibt auch die Kennziffer BIP unverändert, wenn in diesem Bereich mehr oder weniger Wertschöpfung, also Wachstum oder Schrumpfung, erfolgt.

Kommt es zu Verlagerungen zwischen Markt- und privatem Bereich, kann eine reine Betrachtung der BIP-Entwicklung sogar zu völlig falschen Schlüssen führen: Wenn etwa wegen der zunehmenden Berufstätigkeit von Frauen mehr Dienstleistungen (wie in den USA) nachgefragt werden, wächst das BIP, obwohl lediglich eine Verlagerung vom (nicht erfassten) privaten in den (erfassten) Marktbereich stattfindet. Aus ökologischer Sicht wäre dieses Wachstum aber zu begrüßen, weil Essen in der Kantine oder Waschen durch die Wäscherei aufgrund von Mengenvorteilen ${ }^{5}$ tendenziell weniger umweltbelastend ist als die individuelle Essenszubereitung oder das individuelle Waschen. Umgekehrt würde ein Rückzug des Sozialstaates mit Leistungsreduktionen (z. B. Einsparungen im Gesundheitswesen

1 Vgl. World Wide Fund For Nature (WWF) (2012): Living Planet Report 2012. Biodiversität, Biokapazität und neue Wege, Gland.

2 Der ökologische Fußabdruck erfasst alle Ressourcen, die für den jeweiligen Lebensstil einer Person benötigt werden, und zeigt auf, wie viel Fläche erforderlich ist, um die notwendige Energie und die notwendigen Rohstoffe zur Verfügung zu stellen.

3 Hierzu zählen Länder mit mehr als einer Million Einwohner, für die vollständige Daten vorliegen.

4 Vgl. Statistisches Bundesamt/Bundesministerium für Familie, Senioren, Frauen und Jugend (2003): Wo bleibt die Zeit? Die Zeitverwendung der Bevölkerung in Deutschland 2001/02, Berlin/Bonn, S. 13.

5 Es kommt in dem Fall zu sogenannten positiven Skaleneffekten (Massenproduktionsvorteilen), weil bei steigenden Produktionsmengen die eingesetzten Faktoren pro Stück sinken. 
oder bei der Pflege) sich in einem niedrigeren Wachstum, möglicherweise sogar einem zurückgehenden BIP niederschlagen. Konsequent wäre aber aller Voraussicht nach eine Ausweitung unbezahlter Sorgearbeit, um soziale Härten zu vermeiden oder abzumildern. Trotz Rückgang des erfassten Wachstums bzw. sogar des BIP wäre diese Entwicklung aus sozialökologischer Sicht aber keineswegs ein Fortschritt.

(2) Alle Leistungen, die erfasst werden, gehen unterschiedslos in das BIP ein: Der Bau einer Windkraftanlage oder die Einstellung von Lehrern oder Erziehern steigern das BIP genauso wie die Beseitigung von Umweltschäden. Insofern lassen sich auf Grundlage des BIP nur sehr bedingt Aussagen über die Qualität der Entwicklung treffen. Am ehesten ist dies auf niedrigem Entwicklungsniveau möglich, wo ein Anstieg des BIP noch eng mit einer besseren Befriedigung der Grundbedürfnisse (Nahrung, Kleidung, Wohnen) verbunden ist. Die junge ökonomische Glücksforschung hat ergeben, dass in reichen Gesellschaften sich Wachstum und Wohlstand zunehmend entkoppeln: Ab einem gewissen BIP- und damit Einkommensniveau ist im Durchschnitt keine Steigerung des individuellen Wohlbefindens mehr nachweisbar. $^{6}$

(3) Weitere Zweifel an der Aussagekraft des BIP resultieren aus dem Versuch, Preissteigerungen aus der Entwicklung der ökonomischen Leistung herauszurechnen, also die nominelle Steigerung des BIP in eine reale umzurechnen. Reine Preissteigerungen lassen sich solange eindeutig feststellen, wie sich die Qualität der betrachteten Güter nicht verändert. Hat sich aber bei gleichem Preis die Qualität geändert, versuchen die Statistiker dies im Zuge von sogenannten hedonischen Preisbereinigungen zu berücksichtigen, indem sie die höhere (oder niedrigere) Qualität bewerten. Im Ergebnis gehen dann (v. a. elektronische) Produkte, die einem schnellen technologischen Wandel unterliegen, in der Regel mit einem höheren Wert in die Berechnung des BIP ein, als ihr Preis ausweist. Im Ergebnis führt dies wie auch das Statistische Bundesamt festgestellt hat - dazu, dass es allein aufgrund der Anwendung dieser Methodik zu höheren Zuwächsen beim realen BIP kommt. ${ }^{7}$

Damit zeigt sich, dass Forderungen nach Rückgang von BIP und Wachstum als Ausweis einer „richtigen“ ökologischen
Einstellung - jedenfalls soweit sie nicht durch qualitative Vorgaben unterlegt sind - noch sehr im alten BIP-Denken verhaftet sind. Letztlich sind sie nur auf eine Veränderung des Vorzeichens des überkommenen Indikators BIP gerichtet. Notwendige Reflexionen der Erfassungsprobleme wie auch zur Qualität und zu strukturellen Defiziten des Wirtschaftens bleiben ausgeklammert.

Eine gute Illustration bietet die jüngste Eurokrise. Ausgelöst durch Turbulenzen auf den Finanzmärkten kam es zu einem umfassenden Rückgang des BIP im gesamten Euroraum. Die Wirtschaft schrumpfte hier allein im Jahr 2009 um über $4 \%$, in einzelnen Ländern, darunter auch Deutschland, war der Einbruch sogar noch größer. Während Deutschland diesen Rückgang schnell überwinden konnte, schrumpfte vor allem die griechische Wirtschaft drastisch weiter. Bis heute verzeichnet das griechische BIP einen Rückgang um über $20 \%$. Die Folgen für dieses Land sind dramatisch: hohe Arbeitslosigkeit, Verarmung, Verfall der Infrastruktur, Perspektivlosigkeit einer ganzen Generation um nur einige der schlimmsten Folgen zu nennen. Daher würde auch niemand auf die Idee kommen, diesen Verlauf zum Vorbild einer anzustrebenden sozialökologischen Entwicklung zu erklären. ${ }^{8}$

\section{„Degrowth“ - ein vieldeutiger und missverständlicher Begriff}

Vor diesem Hintergrund wird deutlich, dass ein bloßer Wachstumsrückgang oder gar ein sinkendes BIP, also „Negativwachstum“, keine hinreichenden Bedingungen einer wünschenswerten sozialökologischen Transformation sind. Insofern sollte unter der Begriffsneuschöpfung „Degrowth“ - auch wenn es begrifflich naheliegt und offensichtlich vielfach auch so gesehen wird ${ }^{9}$ - nicht einfach Wachstumsrückgang, Negativwachstum oder Schrumpfung des BIP verstanden werden. Die damit verbundene Vorwegnahme des Ergebnisses eines umfassenden Transformationsprozesses - zudem fokussiert auf einen auch noch problematischen Indikator - greift zu kurz, denn Schrumpfung und Expansion sind notwendigerweise Kernbestandteile eines solchen Prozesses. ${ }^{10}$ Bereits in der "Declaration of Degrowth“ der ersten Degrowth-Konferenz 2008 in Paris findet sich der Hinweis, dass der Ansatz ein umfassender sein muss. Neben der Betonung qualitativer Aspekte wie Lebensqualität, Gesundheit, Selbstbestimmung wurde auch
6 Vgl. Ruckriegel, K. (2013): Glücksforschung - Erkenntnisse und Konsequenzen für die Zielsetzung der (Wirtschafts-) Politik, in: Jahrbuch für Nachhaltige Ökonomie 2012/2013, S. $129-147$.

7 Vgl. Lint, S./Eckert, G. (2002): Zur Einführung hedonischer Methoden in der Preisstatistik, in: Wirtschaft und Statistik, Nr. 10, S. 858; Deutscher Bundestag (2013): Enquete-Kommission „Wachstum, Wohlstand, Lebensqualität - Wege zu nachhaltigem Wirtschaften und gesellschaftlichem Fortschritt in der Sozialen Marktwirtschaft", Schlussbericht, Berlin (Bundestags-Drucksache 17/13300), Berlin, S. $131 \mathrm{f}$.
8 Vgl. Brand, U. (2014): Kapitalistisches Wachstum und soziale Herrschaft. Motive, Argumente und Schwächen aktueller Wachstumskritik, in: Prokla 175, S. $289 f$.

9 Vgl. http://www.nachhaltigkeit.info/artikel/degrowth_1849. htm

10 Ulrich Brand betont mit Blick auf die Degrowth-Bewegung: „Es geht ja gerade nicht um ein Ja oder Nein zu Wirtschaftswachstum, sondern um einen genaueren Blick auf die Implikationen, Bedingungen und Konsequenzen kapitalistischen Wachstums." (Brand 2014, a. a. O., S. 301). 
auf die Notwendigkeit zusätzlicher wirtschaftlicher Aktivitäten verwiesen, etwa um die Befriedigung der Grundbedürfnisse aller Menschen zu gewährleisten. ${ }^{11}$ Auch wird es realen Entwicklungen nicht gerecht, wenn „Wachstum“ pauschal mit einer stärkeren Belastung der Umwelt gleichgesetzt würde. Ein forcierter Strukturwandel von der Industrie- zur Dienstleistungsgesellschaft würde beispielsweise mit einer deutlichen Zunahme von Dienstleistungen einhergehen. Da bei Dienstleistungen der Primärenergiebedarf vergleichsweise gering ist, ${ }^{12}$ beinhaltet dieser Strukturwandel auch eine große Chance zu einem insgesamt sinkenden Energieverbrauch.

Vor dem Hintergrund der BIP-Problematik ist es nur folgerichtig, dass im Kontext der Vierten Internationalen Degrowth-Konferenz 2014 in Leipzig auch Probleme der üblichen Messung von Wachstum thematisiert wurden. Auf der Homepage zur Konferenz heißt es, Degrowth stelle auch „das Bruttoinlandsprodukt als zentralen Politikmaßstab infrage. ${ }^{\text {“13 }}$ Sobald aber das BIP als „zentraler Politikmaßstab“ nicht anerkannt wird, kann logischerweise ein weiteres Wachstum des BIP nicht per se verdammt werden. Diese Orientierung findet sich im Ansatz auch bereits in der erwähnten 2008er Degrowth-Deklaration, in der herausgestellt wurde, Degrowth erfordere auch ,increasing economic activity in some cases".

\section{Sozialökologische Transformation statt unqualifizierte Wachstumsförderung}

Trotz der parteienübergreifenden Debatten um Probleme, Grenzen und Sinn des Wachstums beherrscht ein undifferenzierter wachstumsoptimistischer Grundton nach wie vor die Politik. In besonders pointierter Weise vertritt der einflussreiche Magdeburger Ökonom Karl-Heinz Paqué diese Sichtweise. ${ }^{14}$ Wirtschaftliches Wachstum wird von ihm geradezu zur Grundbedingung menschlicher Existenz hochstilisiert, Fortschritt und Entwicklung unisono mit Wachstum gleichgesetzt. Ohne Wachstum sei Entwicklung nicht denkbar. Ein Land, „das sich tatsächlich aus einem Geleitzug des Wachstums ,ausklinkt““, würde „sehr schnell auf ein deutlich niedrigeres Einkommensniveau ab(sacken) “.15
Wachstum sei „der einzige Weg, wie überhaupt im Weltmaßstab die großen Ziele der Menschheit erreicht werden

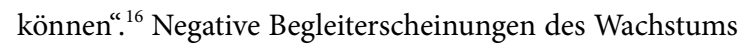
werden nicht einmal zur Kenntnis genommen. Selbst der Ansatz, Wachstum so einzuhegen, dass ein qualitatives Wachstum entstehe, wird zurückgewiesen. Der freie Lauf marktwirtschaftlicher Kräfte sorge automatisch dafür, dass „aus dem quantitativen Wachstum zunehmend qualitatives $^{\text {“17 }}$ werde. Ziel der Politik müsse daher ein möglichst hohes Wachstum sein, das mit der Durchsetzung einer neoliberalen Agenda mit den Kernelementen Liberalisierung, Flexibilisierung und Deregulierung der Wirtschaft zu erreichen sei.

Auch wenn die Ansichten über die Inhalte von Degrowth auseinandergehen und nicht frei von Widersprüchen sind, ist diese Perspektive zweifellos das Gegenteil von Degrowth und sozialökologischer Transformation. Sie ist aber auch das Gegenteil dessen, was Gewerkschaften fordern. Hiermit würde die gegenwärtige verhängnisvolle Entwicklung, was die Ökologie, das Soziale, die Einkommens- und die Vermögensverteilung angeht, nicht nur weitergeschrieben, sondern sogar noch beschleunigt. Eine sozialökologische Transformation setzt demgegenüber eine Debatte über die inhaltliche Bestimmung von Wohlstand voraus.

\section{Wohlstand statt Wachstum als Ziel}

Debatten über die künftige wirtschaftliche und gesellschaftliche Entwicklung müssen zur Kenntnis nehmen, dass alle entwickelten Volkswirtschaften von einem dekadenübergreifenden Trend abnehmender Wachstumsraten des BIP gekennzeichnet sind. ${ }^{18}$ Gesamtwirtschaftliches Wachstum wird hier also - nicht zuletzt auch wegen der demografischen Entwicklung - zukünftig eine geringere Rolle spielen. Bereits in der Vergangenheit hat sich aber gezeigt, dass trotz geringer werdender wirtschaftlicher Zuwächse die Gewinne und die Vermögenseinkommen weiter deutlich gestiegen sind, sodass für die Beschäftigten und die breite Bevölkerung zeitweise sogar reale Einkommensverluste zu verzeichnen waren. Hierdurch hat sich die Spaltung der Gesellschaft weiter verschärft. Insofern stellen zu lösende Vertei-
11 Vgl. http://events.it-sudparis.eu/degrowthconference/en/ Declaration\%20on\%20Degrowth\%20EN.pdf.

12 Bei öffentlichen und privaten Dienstleistungen wird im Vergleich zur Metallerzeugung und -bearbeitung nur ein Vierunddreißigstel an Energie verbraucht, vgl. Statistisches Bundesamt (2013): Umweltnutzung und Wirtschaft. Bericht zu den Umweltökonomischen Gesamtrechnungen, Wiesbaden, S. 50 .

13 http://leipzig.degrowth.org/de/selbstverstandnis/.

14 Karl-Heinz Paqué war u. a. von 2002 bis 2006 Finanzminister des Landes Sachsen-Anhalt, von 2011 bis 2013 sachverständiges Mitglied der Enquete-Kommission
„Wachstum, Wohlstand, Lebensqualität“ des Deutschen Bundestages.

15 Paqué, K.-H. (2010): Wachstum! Die Zukunft des globalen Kapitalismus, München, S. 30.

16 Ebd. S. 1.

17 Ebd. S. 25.

18 Vgl. Afheldt, H. (1994): Wohlstand für Niemand? Die Marktwirtschaft entlässt ihre Kinder, Frankfurt a. M./Wien; Reuter, N. (2010): Der Arbeitsmarkt im Spannungsfeld von Wachstum, Ökologie und Verteilung, in: Seidl, I./Zahrnt, A. (Hrsg.): Postwachstumsgesellschaft, Marburg, S. 89f. 
lungsprobleme eine weitere große Herausforderung einer sozial-ökologischen Transformation dar.

Für die Opfer der letzten Krise - die südlichen Euroländer - stellt sich zunächst die vordringliche Aufgabe, notwendige wirtschaftliche Zuwächse zur Befriedigung grundlegender Bedürfnisse nach Arbeit, Nahrung, Kleidung, Wohnung, Bildung mit einem umfassenden ökologischen Umbau zu verbinden. Aus diesem Grunde fordert der Deutsche Gewerkschaftsbund (DGB) im Rahmen eines europäischen „Marshall-Plans“ umfassende Investitionen in nachhaltige Energieerzeugung, in die Reduktion des Energieverbrauchs, in nachhaltige Industrien und Dienstleistungen, in Bildung und Ausbildung, in Forschung und Entwicklung, in moderne Verkehrsinfrastruktur, emissionsarme Städte und Gemeinden und in die Effizienz der öffentlichen Verwaltungen. ${ }^{19}$ Damit sind konkrete Aufgabenfelder umschrieben, die Arbeit und Einkommen schaffen und sich zweifellos auch erst einmal - vor allem in den südlichen Krisenländern - wieder in einem höheren Wachstum des BIP niederschlagen. Ziel wäre aber etwa die Reduktion von Brennstoffimporten, deren Umfang nach Berechnungen der Europäischen Kommission jährlich um bis zu $300 \mathrm{Mrd}$. $€$ fallen würde - was nicht nur die Umwelt, sondern auch die nationalen Haushalte entlasten würde. ${ }^{20}$ Insofern sind Marshall-Plan und eine Degrowth-Orientierung auch keine Gegensätze. Der Marshall-Plan der Gewerkschaften kann sogar als ein Beispiel von „increasing economic activity in some cases“ im Sinne der Degrowth-Strategie gesehen werden. Ziel ist der sozialökologische Umbau, mit Blick auf die südlichen Krisenländer ist es zweifellos aber erst einmal auch ein sozialökologischer Aufbau.

Vor dem Hintergrund der krisenübergreifenden grundlegenden Wachstumsabschwächung muss es langfristiges Ziel und Strategie von Politik sein, Wohlstand unter niedrigen bzw. weiter sinkenden Wachstumsraten zu sichern und weiter zu steigern. Allerdings scheinen sich die Bestimmungsfaktoren von Wohlstand zu verändern. Es wurde schon darauf hingewiesen, dass im Zuge der Industrialisierung sich Wachstum und Wohlstand offensichtlich zunächst parallel, dann aber zunehmend aus- bzw. sogar gegeneinander entwickeln: Mit der Steigerung von Produktivität und Wirtschaftsleistung nehmen grundsätzlich die Möglichkeiten $\mathrm{zu}$, mehr Waren und Dienstleistungen zu nutzen. Je niedriger das Einkommens- und Produktivitätsniveau ist, desto höher ist bei einer Steigerung des BIP auch tenden- ziell der Wohlstandsgewinn. Solange die grundlegenden Bedürfnisse der Menschen nicht ausreichend befriedigt sind, bedeutet ein Mehr an dafür notwendigen Gütern eine klare Wohlstandssteigerung. Deshalb spielt in den südlichen Ländern das wirtschaftliche Wachstum auf absehbare Zeit auch noch bzw. wieder eine größere Rolle als hierzulande. Mit zunehmender Befriedigung grundlegender Bedürfnisse sinkt allerdings der Nutzen zusätzlicher Waren und Dienstleistungen, damit auch die Bedeutung weiteren quantitativen Wachstums. Gleichzeitig nehmen die negativen Begleiterscheinungen des Wachstums zu, die sich einerseits in schlechterer Umweltqualität, sinkender Artenvielfalt, steigender Ressourcenknappheit, andererseits in wachsendem Arbeits- und Leistungsdruck und ausufernden Arbeitszeiten zeigen. ${ }^{21}$ Ein „Weiter so“ führt dann nicht nur zu massiven ökologischen Problemen, sondern bringt per saldo auch kaum noch eine Wohlstandssteigerung mit sich. Diese Entwicklung verschärft sich noch einmal, wenn große Bevölkerungsteile wegen einer zunehmenden Ungleichverteilung von Einkommen und Vermögen noch nicht einmal von Einkommenssteigerungen profitieren.

\section{Sozialökologische Transformation}

Eine Fokussierung auf qualitativer Entwicklung statt (quantitativem) Wachstum ist offensichtlich unumgänglich. Hier liegt auch der eigentliche Kern der Degrowth-Strategie. Vor allem darf eine bloße Steigerung des BIP kein Ziel der Wirtschaftspolitik mehr sein. In der Vergangenheit wurde genau dies aber immer wieder durch auf Deregulierung, Flexibilisierung und Liberalisierung zielende politische Maßnahmen zwecks Befreiung wachstumstreibender Marktkräfte versucht. ${ }^{22}$ Auf solche Maßnahmen, die auf ein stetiges Wachstum abzielen, ist die Politik in Deutschland sogar verpflichtet. So steht es immer noch im „Stabilitäts- und Wachstumsgesetz" aus dem Jahr 1967, das das Staatsziel des gesamtwirtschaftlichen Gleichgewichts in Artikel 109 des Grundgesetzes konkretisiert.

Deshalb wäre dieses Gesetz schnellstmöglich in ein „Stabilitäts- und Entwicklungsgesetz“ bzw. „Wohlstands- und Nachhaltigkeitsgesetz" umzuformulieren. Sobald nicht mehr Wachstum, sondern eine wohlstandssteigernde Entwicklung das Ziel ist, ergäbe sich die Notwendigkeit, die Bedingungen einer solchen Entwicklung in einem demokratischen Prozess zu definieren („Welche Gesellschaft wol-
19 Vgl. http://www.dgb.de/themen/++co++985b632e-407e11e2-b652-00188b4dc422.

20 Vgl. Spencer, T. et al. (2012): "Grüne“ Investitionen in einem europäischen Wachstumspaket, in: DIW Wochenbericht 79 (25), S. 4f.; Hirschhausen, C. v. et al. (2014): Europäische Energiewirtschaft: Hoher Investitionsbedarf für Nachhaltigkeit und Versorgungssicherheit, in: DIW Wochenbericht 27/2014, S. 661-666.
21 Vgl. Reuter, N. (2014): Wohlstand ohne Wachstum?, http://www2.alternative-wirtschaftspolitik.de/uploads/ m1314.pdf; Wilkinson, R./Pickett, K. (2010): Gleichheit ist Glück, Berlin, S. 20.

22 Ein Beispiel ist das sogenannte „Gesetz zur Beschleunigung des Wirtschaftswachstums (Wachstumsbeschleunigungsgesetz)“. Es wurde Ende 2009 vom Deutschen Bundestag verabschiedet. Mit ihm wurden steuerliche Sofortmaßnahmen umgesetzt, die wieder zu mehr Wachstum führen sollten. 
len wir?"). ${ }^{23}$ Eine Rückführung der massiven Ungleichverteilung, die Stärkung des Sozialstaats, die Verbesserung der Arbeitsbedingungen, der Abbau der nach wie vor bestehenden Massenarbeitslosigkeit, die Festlegung von Grenzen für den Ressourcenverbrauch und $\mathrm{CO}_{2}$-Ausstoß, aber auch die Stärkung von Effizienz (höhere Ressourcenproduktivität, Dematerialisierung), Konsistenz (Naturverträglichkeit, Qualität) und Suffizienz (Selbstbegrenzung, Maßhalten) würden ganz oben auf der politischen Agenda stehen. Und mit Blick auf diese inhaltlichen Anforderungen würde herkömmliches BIP-Wachstum allenfalls ein Mittel sein, das in unterschiedlichen Ländern zu unterschiedlichen Zeiten eine unterschiedliche Bedeutung hätte.

In einem zweiten Schritt müssten dann die notwendigen politischen Maßnahmen („Leitplanken“) diskutiert und festgelegt werden, mit denen die verabredeten Ziele erreicht werden können. Während dies vor allem für Südeuropa der erwähnte Marshallplan sein könnte, würde dies bei uns etwa eine alternative Steuer- und Abgabenpolitik zur Bekämpfung der wachsenden Verteilungsungleichheit und zur Stärkung der Energiewende bedeuten, eine andere Arbeitsmarktpolitik für mehr gute Arbeit statt Billigjobs oder

23 Auch Ulrich Brand unterstreicht die Bedeutung demokratischer Prozesse als Grundlage eines sozialökologischen Transformationsprozesses, vgl. Brand (2014), a.a. O., S. $403 f$.

24 Vgl. Jackson, T. (2011): Wohlstand ohne Wachstum. Leben und Wirtschaften in einer endlichen Welt, München, S. 177ff.

25 Vgl. zur Indikatorendebatte umfassend Deutscher Bundestag (2013), a.a.O., S. 231-351. höhere öffentliche Ausgaben für eine bessere Bildung und Gesundheitsversorgung. ${ }^{24}$

Eine derartige demokratische Gestaltung würde zu einem neuen Wirtschaften führen. Einzelne Bereiche müssten zweifellos wachsen, andere schrumpfen. Zum Beispiel würde ein wachsender öffentlicher Personennahverkehr (ÖPNV) einem schrumpfenden Individualverkehr gegenüberstehen oder einer umfassenden Gebäudedämmung ein sinkender Energieeinsatz. Diese inhaltliche Qualifizierung der wirtschaftlichen Entwicklung, deren Erfolg selbstverständlich anhand neuer Indikatoren gemessen werden müsste, würde die fragwürdige Orientierung am Indikator BIP überwinden. ${ }^{25} \mathrm{Ob}$ Letzterer dann Wachstum oder Schrumpfung ausweist, wäre nicht mehr die Frage - und auch unerheblich. Vielleicht sollte dann auch der Begriff des Degrowth, der stark auf die überkommene Kategorie des BIP bezogen ist, einer mehr inhaltlich bestimmten Bezeichnung Platz machen. Einstweilen würde sich hierfür der Begriff der sozialökologischen Transformation anbieten.

\section{AUTOR}

NORBERT REUTER, PD Dr., ist Ökonom im Bereich Wirtschaftspolitik beim Bundesvorstand der Vereinten Dienstleistungsgewerkschaft (ver.di), lehrt Volkswirtschaftslehre an der RheinischWestfälischen Technischen Hochschule (RWTH) Aachen und war von 2011 bis 2013 sachverständiges Mitglied der Enquete-Kommission „Wachstum, Wohlstand, Lebensqualität" des Deutschen Bundestages. Arbeitsschwerpunkte: Strukturwandel, Verteilung, demografische Entwicklung.

norbert.reuter@verdi.de 